\title{
Synthesis of the new ring systems indeno[1,2-d]pyrimidinones, indeno[1,2-e]pyrrolo[1,2-a]pyrimidinones and indeno[1,2-e]pyrimido[1,2-a]izoindoles
}

\author{
Márta Palkó, Máté Dervarics, and Ferenc Fülöp* \\ Institute of Pharmaceutical Chemistry, University of Szeged, H-6701, POB 121, Hungary \\ E-mail: fulop@pharm.u-szeged.hu
}

Dedicated to Professor Eusebio Juaristi on his $55^{\text {th }}$ birthday

(received 17 Jun 05; accepted 20 Aug 05; published on the web 23 Aug 05)

\begin{abstract}
Indeno[1,2-d]pyrimidinones, indeno[1,2-e]pyrrolo[1,2-a]pyrimidinones and indeno[1,2e]pyrimido[1,2-a]izoindoles were prepared as new ring systems by ring enlargement of azetidinone 2 and ring closure of amino ester $\mathbf{3}$ and 1,3-diamine 5. These ring closures resulted in pure diastereomers, the stereochemistry of which was determined by NMR spectroscopy.
\end{abstract}

Keywords: Ring closure, ring enlargement, diastereoselective, NMR spectroscopy

\section{Introduction}

In the past two decades, a number of cyclic $\beta$-amino acid derivatives have been synthesized. Some of them have useful pharmacological effects, ${ }^{1}$ and they are widely used for the preparation of saturated 1,3-heterocycles. The synthesis and stereochemical aspects have been thoroughly studied for the cis- or trans-cyclohexane-, -cyclohexene-, and diexo- and diendo-fused norbornane- and norbornene-1,3-heterocycles. ${ }^{2}$ To date, only few indane-fused heterocycles have been prepared. ${ }^{3-10}$ Because of their therapeutic interest, the syntheses of cycloalkane-fused pyrimidinones have been studied, ${ }^{2}$ but syntheses of their indane-condensed derivatives have not yet been reported.

Our present aim was to prepare new cis-1-aminoindane-2-carboxylic acid derivatives and to synthesize some indane-fused pyrimidines. 


\section{Results and Discussion}

\section{Syntheses}

Racemic 3,4-benzo-6-azabicyclo[3.2.0]heptan-7-one (2) and ethyl cis-1-aminoindane-2carboxylate (3) were prepared from indene by chlorosulfonyl isocyanate addition, followed by hydrolysis and ring opening. ${ }^{11}$ Compounds $\mathbf{2}$ and $\mathbf{3}$ can be used as starting substances for the preparation of other bifunctional compounds, e. g. 1,3-aminoalcohols and 1,3-diamines. When amino ester 3 was reacted with $\mathrm{MeNH}_{2}$, a mixture of cis- and trans-aminocarboxamides (cis:trans $=1: 1$ ) was formed. This is in accordance with our earlier results: isomerization was observed in the amidation of ethyl cis-2-aminocyclopentanecarboxylate. ${ }^{12}$ For the preparation of cis-aminocarboxamide 4, azetidinone 2 was activated with a Boc group. The reaction of the $N$ Boc derivative of 2 with $\mathrm{MeNH}_{2}$ for $2 \mathrm{~h}$ at $4{ }^{\circ} \mathrm{C}$, resulted in the protected cis-carboxamide. The Boc group was easily removed with $\mathrm{HCl}$ in dry $\mathrm{EtOH}$ at room temperature, which afforded the hydrochloride of 4. cis-2-Methylaminomethylindan-1-ylamine (5) was prepared by $\mathrm{LiAlH}_{4}$ reduction of 4 (Scheme 1).

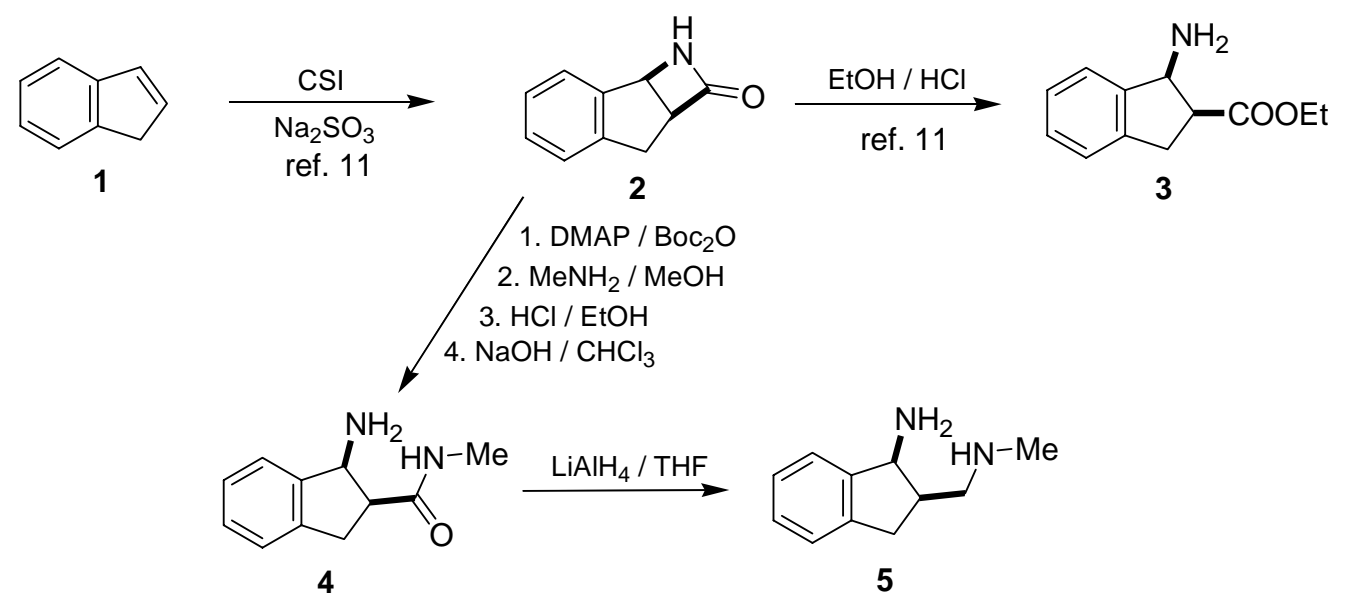

\section{Scheme 1}

The indane-condensed pyrimidinones 6-10 were prepared by ring enlargement ${ }^{2,13}$ of azetidinone 2 with the corresponding imidates or lactim ethers. When 2 was melted with imidates or lactim ethers at $150{ }^{\circ} \mathrm{C}$ for $8 \mathrm{~h}$, the desired pyrimidinones 6-10, were formed. The first step in the reaction is the splitting-off of alcohol, resulting in an amidine intermediate which, after transamidation, yields the ring enlargement products (Scheme 2). 

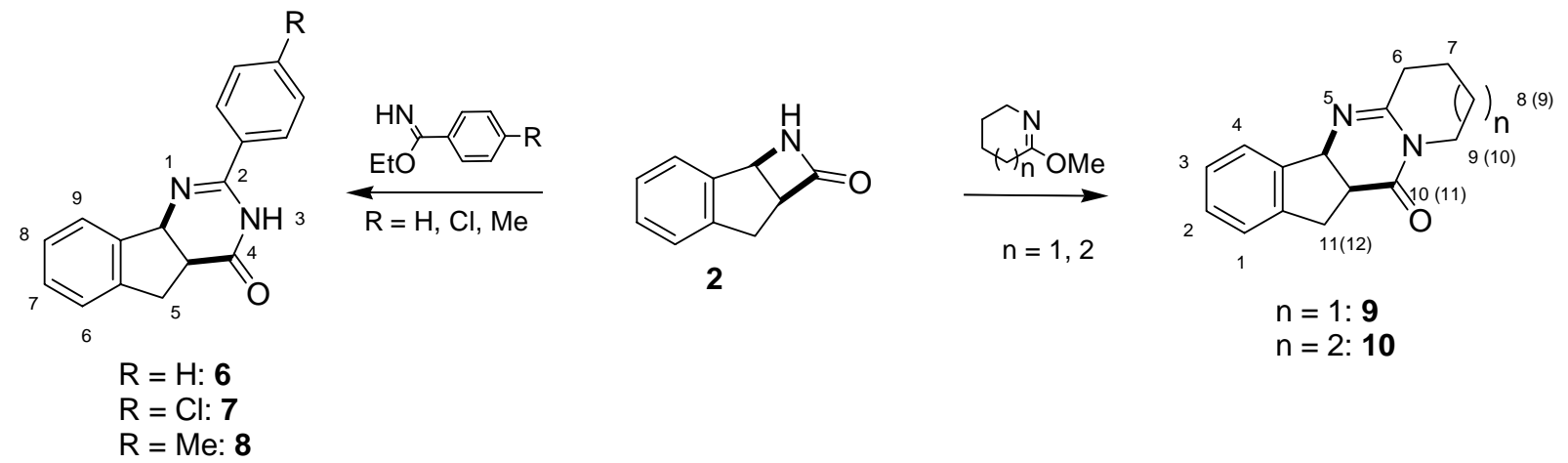

\section{Scheme 2}

When amino ester 3 was reacted with one equivalent of $p$-chlorobenzaldehyde in toluene at room temperature Schiff base 11 was formed. This was treated with $\mathrm{NH}_{3}$ or $\mathrm{MeNH}_{2}$ in $\mathrm{MeOH}$ solution to afford a mixture of indeno[1,2-a]pyrimidin-4-one C-2 epimers $(\mathbf{1 2}, 2: 1 ; \mathbf{1 3}, 3: 1)$; fractional crystallization furnished the major diastereomers.

On reaction with various isocyanates and isothiocyanates, 3 yielded the urea and thiourea derivatives 14a-d. When attempts were made to cyclize 14a-d to pyrimidinones, only $N$ methylthiourea 14b was cyclized successfully to pyrimidinone 15 in EtOH containing 22\% dry $\mathrm{HCl}$. Under the same conditions, the other derivatives gave the elimination product indene-2carboxylic acid (16) (Scheme 3).

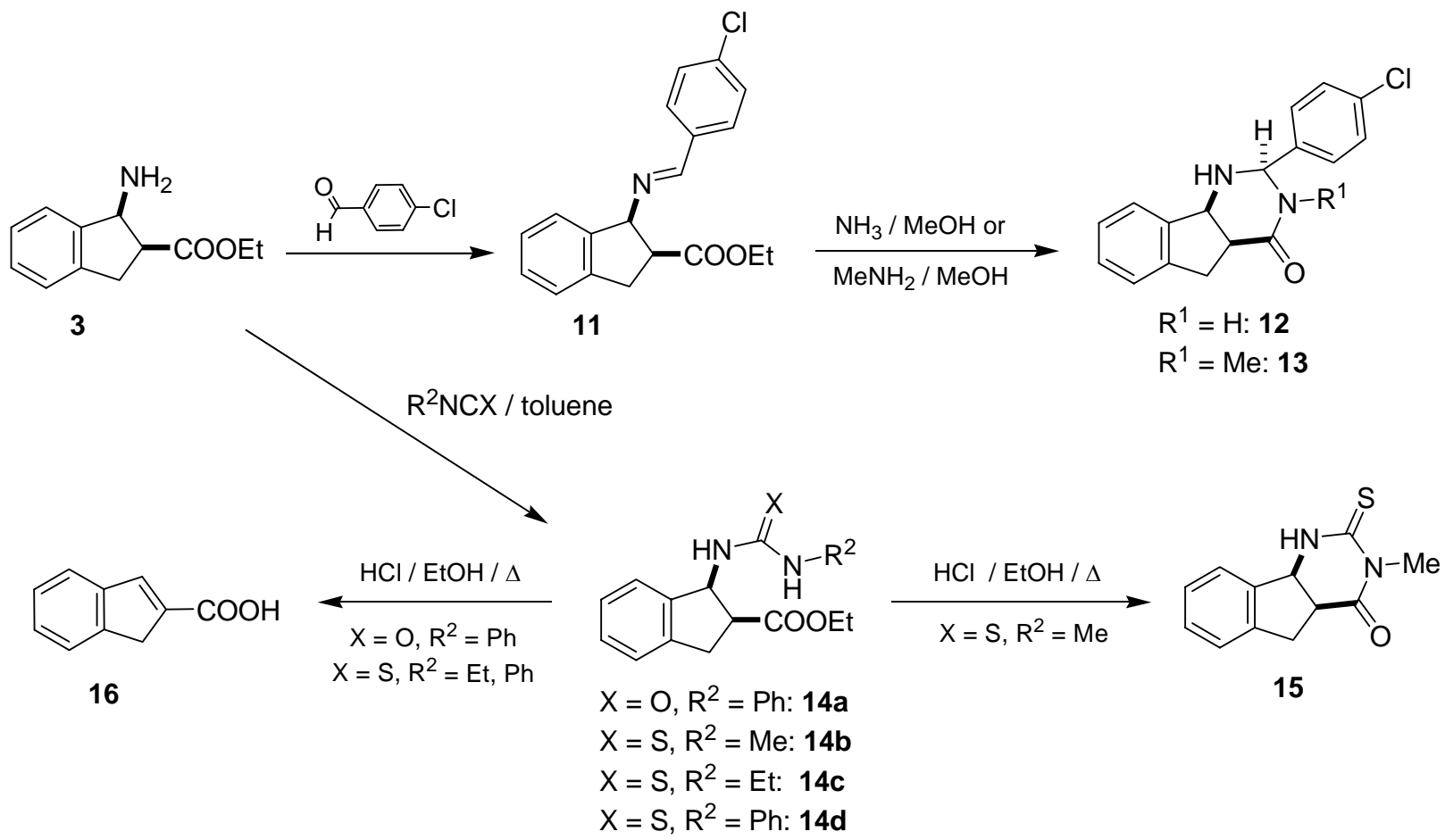

Scheme 3 
When $\mathrm{N}$-methyldiamine 5 was reacted with levulinic acid or 2-formylbenzoic acid in boiling toluene, penta- and tetracycles 19 and 22 were formed in good yields. NMR measurements indicated that 19 and 22 were formed with excellent diastereoselectivity (de $100 \%$ ) with the relative configurations depicted in Scheme 4 . The ring closures of 5 can be categorized as domino reactions. ${ }^{14}$ The intermediates $\mathbf{1 7}$ and $\mathbf{2 0}$ possess a ring-chain tautomeric character, the second ring closure step causing a shift in the tautomeric equilibrium. The high diastereoselectivity can be explained as a result of the kinetic control governing the second ring closure. $^{15-17}$

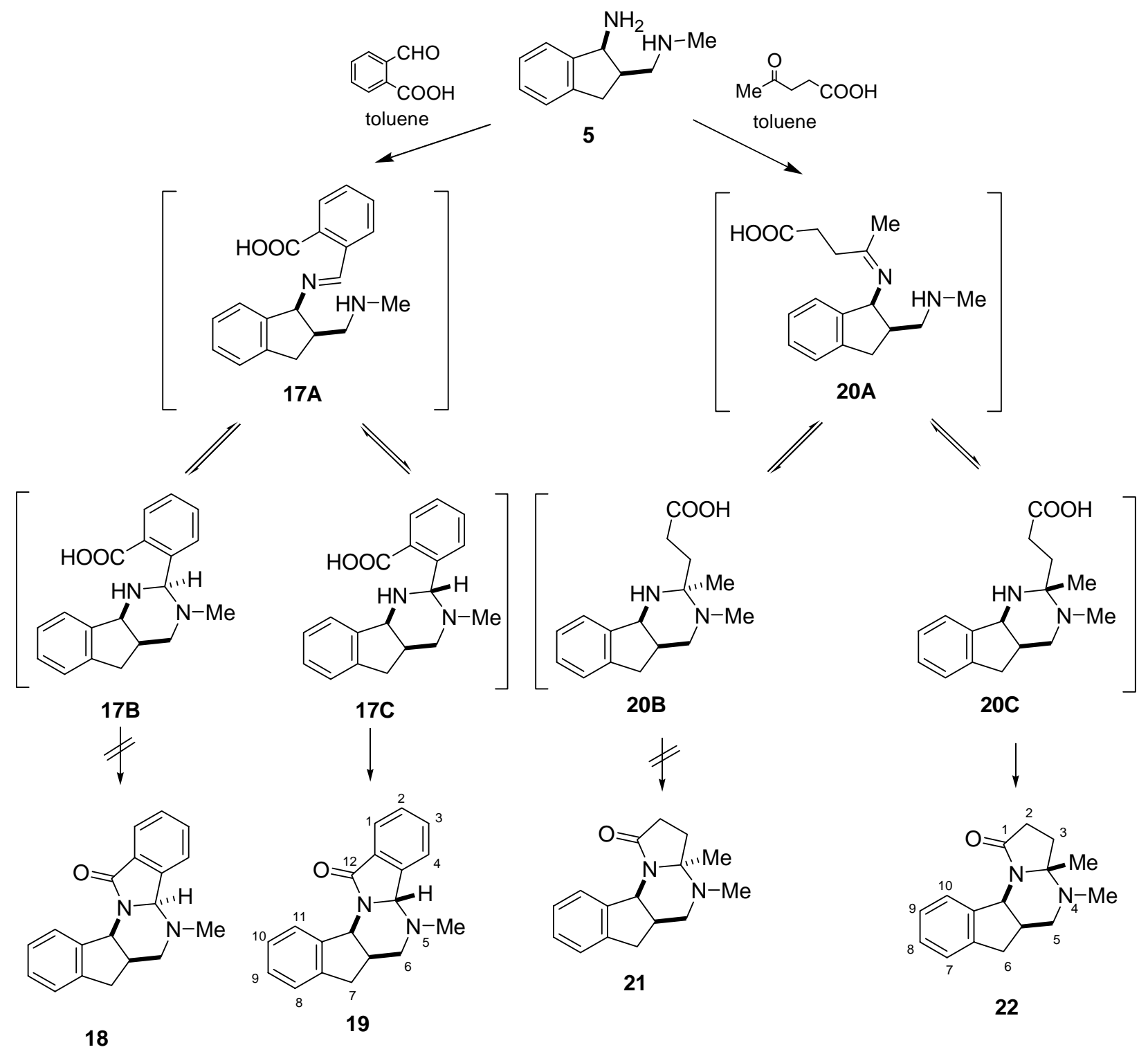

\section{Scheme 4}


Diamine 5 was condensed in $\mathrm{MeOH}$ with seven aromatic aldehydes with different electronic characters. The reaction reached completion in a few hours, even at room temperature. After evaporation and purification, well-defined products $\mathbf{2 3 a - g}$ were obtained. The ${ }^{1} \mathrm{H}$ NMR spectra clearly proved that these derivatives exist as ring-ring epimers. The ratios of the two ring forms were determined by integration of the well-separated N-CHAr-N (ring) singlets. The epimeric rates were practically constant $(\mathbf{2 3 B}: \mathbf{2 3 C}=6: 4)$, independently of the electronic character of the aryl substituent.

These results are in accordance with those of our previous studies, because some of the $N$ substituted hexahydropyrimidinones and tetrahydroquinazolinones proved to be ring-chain tautomeric mixtures in $\mathrm{CDCl}_{3}$ at $300 \mathrm{~K}$, whereas the $\mathrm{N}$-methylhexahydropyrimidine and $\mathrm{N}$ methyltetrahydroquinazoline derivatives exist solely as ring forms. ${ }^{14,18}$

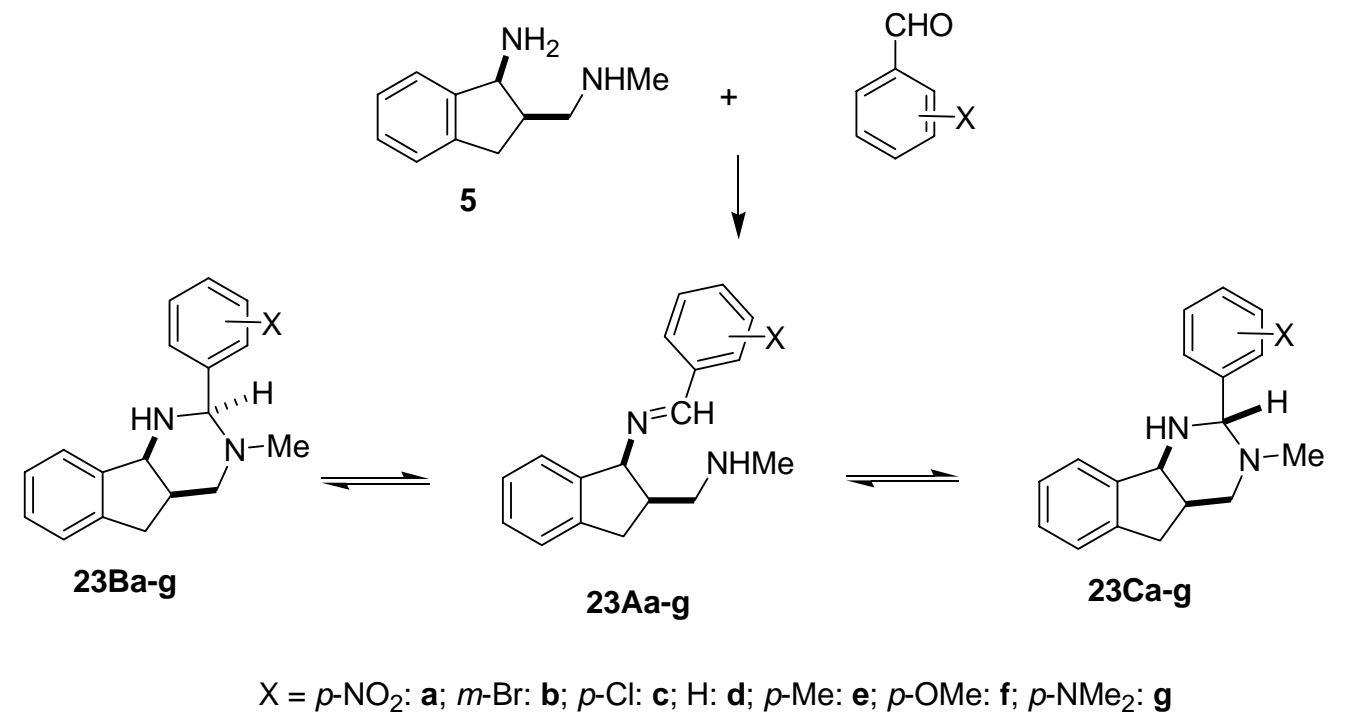

\section{Scheme 5}

\section{NMR spectroscopy}

For 12 and 13, the relative orientation of the 2-aryl substituent was determined by using characteristic NOE interactions. The clear NOESY cross-peak between H-2 and H-9b in 12 indicates a trans relationship for 2-aryl and $\mathrm{H}-9 \mathrm{~b}$. As regards $\mathbf{1 8}$ or $\mathbf{1 9}$, the relative spatiality of $\mathrm{H}-4 \mathrm{~b}$ was determined by modelling and consideration of the NOE interactions. The rigid structures show that in $\mathbf{1 8}$ significant NOE cross-talk should be observed between $\mathrm{H}-4 \mathrm{~b}$ and $\mathrm{H}$ $11 \mathrm{~b}$, but this is not detected in the NOESY spectrum. All the other spectral parameters accord well with structure 19. In 21 or 22, an NOE signal can be detected from Me-3a to H-5ax. H-5ax exhibits a vicinal coupling ca. of $11 \mathrm{~Hz}$ and a weak NOESY cross-peak to H-5a. These strongly 
support Me-3 and H-5a being situated on opposite sides of the heterocyclic ring, indicating structure 22.

In the spectra of $\mathbf{2 3}$, we found no sign of the azomethine proton of the open form. The proportions of the two ring forms were calculated from the integrals of the H-2 signals. The chemical shifts of $\mathrm{H}-2$ of ring forms $\mathrm{B}$ and $\mathrm{C}$ were assigned via the NOESY NMR. The H-2 signal at around $\delta 4.2 \mathrm{ppm}$ has an NOE cross-peak with $\mathrm{H}-9 \mathrm{~b}$, indicating the cis orientation (ring form B), while the H-2 signal at around $\delta 4.6$ ppm has no NOE cross-peak with H-9b, indicating the trans orientation of these protons (ring form C).

\section{Experimental Section}

General Procedures. Melting points were determined with a Koffler apparatus and are not corrected. Elemental analyses were performed with a Perkin-Elmer 2400 CHNS instrument. Merck Kieselgel 60F254 plates were used for TLC: the eluent was toluene-MeOH 4:1. MS data were obtained with a Finnigan Mat 95S spectrometer in EI mode. Column chromatography was performed on silica gel (Merck 60, 70-230 mesh). ${ }^{1} \mathrm{H}$ NMR and ${ }^{13} \mathrm{C}$ NMR spectra were recorded in $\mathrm{CDCl}_{3}$ solution in $5 \mathrm{~mm}$ tubes, at room temperature, on a Bruker DRX 400 spectrometer at $400.13\left({ }^{1} \mathrm{H}\right)$ and $100.61\left({ }^{13} \mathrm{C}\right) \mathrm{MHz}$, with the deuterium signal of the solvent as the lock and TMS as internal standard. For the equilibria to be established in the tautomeric compounds, samples were dissolved in $\mathrm{CDCl}_{3}$ and the solutions were allowed to stand at ambient temperature for 1 day before the ${ }^{1} \mathrm{H}$ NMR spectra were run. The number of scans was usually 64. 3,4-Benzo-6azabicyclo[3.2.0]heptan-7-one (2) and ethyl cis-1-aminoindane-2-carboxylate (3) were prepared according to reported methods. ${ }^{11}$

cis-1-Aminoindane-2-carboxylic acid methylamide (4). 3,4-Benzo-6-azabicyclo[3.2.0]heptan7-one $(2 ; 4.7 \mathrm{~g}, 0.03 \mathrm{~mol})$ was dissolved in $20 \mathrm{ml}$ dry $\mathrm{CH}_{2} \mathrm{Cl}_{2}$. The solution was stirred, and $0.37 \mathrm{~g}$ 4-dimethylaminopyridine (DMAP) was added, followed by di-tert-butyldicarbonate $(7.2 \mathrm{~g}, 0.033 \mathrm{~mol})$. After stirring at room temperature for $5 \mathrm{~h}$, the reaction mixture was left to stand overnight. The brownish reaction mixture was then extracted with brine and dried $\left(\mathrm{Na}_{2} \mathrm{SO}_{4}\right)$. After evaporation, 7.1 g 3,4-benzo-6-tert-butoxycarbonyl-6-azabicyclo[3.2.0]heptan7 -one was obtained, as a white crystalline product. The crude protected $\beta$-lactam was dissolved in $60 \mathrm{ml}$ of a $40 \%$ solution of $\mathrm{MeNH}_{2}$ in dry $\mathrm{MeOH}$. The reaction mixture was allowed to stand at $4{ }^{\circ} \mathrm{C}$ for $2 \mathrm{~h}$ and was next evaporated, first at room temperature and then on a $60{ }^{\circ} \mathrm{C}$ water bath. A white crystalline powder was obtained. The protected cis-aminocarboxamide hydrochloride was dissolved in $20 \mathrm{ml} 3 \mathrm{M} \mathrm{HCl}$ solution in $\mathrm{EtOH}$. For removal of the protecting group, the reaction mixture was left to stand at room temperature for $2 \mathrm{~h}$ and then evaporated at room temperature. After evaporation, $4.2 \mathrm{~g} 4$ hydrochloride was obtained, which was purified by recrystallization from EtOH. Amide base 4 was obtained from the hydrochloride by alkaline treatment $(10 \% \mathrm{NaOH})$, extraction $\left(\mathrm{CH}_{2} \mathrm{Cl}_{2}\right)$, and evaporation under reduced pressure. 
4. $\mathrm{HCl}$ : yield $3.7 \mathrm{~g}, 63 \%$, mp 252-254 ${ }^{\circ} \mathrm{C}$. Anal. Calcd. for $\mathrm{C}_{11} \mathrm{H}_{15} \mathrm{ClN}_{2} \mathrm{O}$ (226.75): $\mathrm{C}, 58.21 ; \mathrm{H}$, 6.61; Cl, 15.66; N, 12.35. Found: C, 58.43; H, 6.87; N, 15.37. NMR data: ${ }^{1} \mathrm{H}$ NMR $\delta: 2.874$ (3H, s, $\left.\mathrm{CH}_{3}\right), 3.34-3.46(2 \mathrm{H}, \mathrm{m}, \mathrm{H}-3), 3.65(1 \mathrm{H}, \mathrm{dd}, J=15.1,7.1 \mathrm{~Hz}, \mathrm{H}-2), 5.05(1 \mathrm{H}, \mathrm{d}, J=7.1 \mathrm{~Hz}, \mathrm{H}-$ 1), 7.44-7.55 (3H, m, H-5, H-6, H-7), $7.6(1 \mathrm{H}, \mathrm{d}, J=7.3 \mathrm{~Hz}, \mathrm{H}-4) .{ }^{13} \mathrm{C}$ NMR $\delta: 26.39,34.39$, $46.46,56.59,125.42,125.83,128.18,130.83,137.19,142.89,173.94$.

cis-2-Methylaminomethylindane-1-ylamine (5). To a stirred suspension of $\mathrm{LiAlH}_{4}(1 \mathrm{~g}$, $26 \mathrm{mmol})$ in $60 \mathrm{ml}$ dry THF was added a solution of 4 amide ( $2 \mathrm{~g}$, in $10 \mathrm{mmol}$ dry THF). The resulting suspension was refluxed for $4 \mathrm{~h}$ and then decomposed by the addition of a mixture of $2 \mathrm{ml}$ water and $10 \mathrm{ml}$ THF. The inorganic material was filtered off and washed with THF $(3 \mathrm{x}$ $20 \mathrm{ml}$ ). After filtration, the solvent was evaporated off to give an oil, which was dissolved in EtOH $(20 \mathrm{ml})$ and converted to the crystalline hydrochloride of 5 with $20 \% \mathrm{HCl}$ in $\mathrm{EtOH}(2 \mathrm{ml})$ and $\mathrm{Et}_{2} \mathrm{O}(50 \mathrm{ml})$. The crystals were filtered off and recrystallized from $\mathrm{MeOH}-\mathrm{Et}_{2} \mathrm{O}$. Pure diamine base 5 was obtained from the hydrochloride by alkaline treatment $(10 \% \mathrm{NaOH})$, extraction $\left(\mathrm{CH}_{2} \mathrm{Cl}_{2}\right)$, and evaporation under reduced pressure. 5. $\mathrm{HCl}$ : yield $1.49 \mathrm{~g}, 70 \%, \mathrm{mp} 250$ $255^{\circ} \mathrm{C}$. Anal. Calcd. for $\mathrm{C}_{11} \mathrm{H}_{17} \mathrm{ClN}_{2}$ (212.73): $\mathrm{C}, 62.05$; $\mathrm{H}, 7.99 ; \mathrm{Cl}, 16.69 ; \mathrm{N}, 13.16$. Found: $\mathrm{C}$, 61.93; H, 7.82; Cl, 16.39; N, 13.32. NMR data: ${ }^{1} \mathrm{H}$ NMR $\delta: 2.93\left(3 \mathrm{H}, \mathrm{s}, \mathrm{CH}_{3}\right), 3.19-3.29(2 \mathrm{H}, \mathrm{m}$, $\mathrm{CH}_{2} \mathrm{~N}, 3.33-3.43(2 \mathrm{H}, \mathrm{m}, \mathrm{H}-3), 3.58(1 \mathrm{H}, \mathrm{dd}, J=12.5,4.7 \mathrm{~Hz}, \mathrm{H}-2), 5.03(1 \mathrm{H}, \mathrm{d}, J=6.3 \mathrm{~Hz}, \mathrm{H}-$ 1), 7.45-7.58 (3H, m, H-4, H-5, H-6), 7.62 (1H, d, $J=7.5 \mathrm{~Hz}, \mathrm{H}-7) .{ }^{13} \mathrm{C}$ NMR $\delta: 34.4,40.29$, $48.1,50.06,57.87,125.32,125.72,127.12,130.14,137.95,143.81$.

\section{General procedure for the preparation of tetrahydroindeno[1,2-d]pyrimidin-4-ones 6-8}

A mixture of azetidinone $8(0.5 \mathrm{~g}, 3.14 \mathrm{mmol})$ and the corresponding ethyl benzimidate $(3.14 \mathrm{mmol})$ was kept at $150-160{ }^{\circ} \mathrm{C}$ for $8 \mathrm{~h}$. The end of the reaction was detected by means of TLC. After cooling, the products were recrystallized from EtOH.

$\left(4 \mathrm{a} S^{*}, 9 \mathrm{bS} *\right)$-2-Phenyl-3,4a,5,9b-tetrahydroindeno[1,2-d]pyrimidin-4-one (6). yield $0.68 \mathrm{~g}$, $83 \%$, mp 231-232 ${ }^{\circ} \mathrm{C}$. Anal. Calcd. for $\mathrm{C}_{17} \mathrm{H}_{14} \mathrm{~N}_{2} \mathrm{O}$ (262.31): C, 77.84; H, 5.38; N, 10.68. Found: C, 77.67; H, 5.42; N, 10.93. NMR data: ${ }^{1} \mathrm{H}$ NMR $\delta: 3.28-3.35(2 \mathrm{H}, \mathrm{m}, \mathrm{H}-5), 3.55(1 \mathrm{H}, \mathrm{dt}, J=$ $12.0 \mathrm{~Hz}, 7.1 \mathrm{~Hz}, \mathrm{H}-4 \mathrm{a}), 5.52(1 \mathrm{H}, \mathrm{d}, J=7.1 \mathrm{~Hz}, \mathrm{H}-9 \mathrm{~b}), 7.23-7.25$ (3H, m, H-7, H-8, H-9), 7.42$7.49(3 \mathrm{H}, \mathrm{m}, m-\mathrm{Ph}, p-\mathrm{Ph}), 7.52(1 \mathrm{H}, \mathrm{d}, J=5.8 \mathrm{~Hz}, \mathrm{H}-6), 7.78(2 \mathrm{H}, \mathrm{d}, J=7.1 \mathrm{~Hz}, o-\mathrm{Ph}) 8.47(1 \mathrm{H}$, bs, NH). ${ }^{13} \mathrm{C}$ NMR $\delta: 35.10,42.60,64.80,124.90,124.97,126.78,127.74,128.45,129.23$, $131.6,134.12,140.37,143.65,172.55,176.57$.

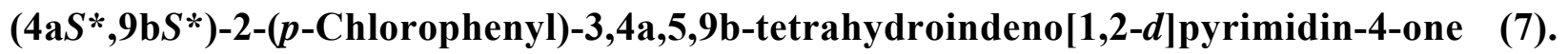
yield $0.75 \mathrm{~g}, 81 \%$, mp 205-210 ${ }^{\circ} \mathrm{C}$. Anal. Calcd. for $\mathrm{C}_{17} \mathrm{H}_{13} \mathrm{ClN}_{2} \mathrm{O}$ (296.76): C, 68.81; H, 4.42; $\mathrm{Cl}, 11.95$; N, 9.44. Found: C, 69.11; H, 4.32; Cl, 11.83; N, 9.23. NMR data: ${ }^{1} \mathrm{H}$ NMR $\delta: 3.28-$ 3.35 (2H, m, H-5), 3.54 (1H, dt, $J=12 \mathrm{~Hz}, 7.5 \mathrm{~Hz}, \mathrm{H}-4 \mathrm{a}), 5.52$ (1H, d, J=7.5 Hz, H-9b), 7.24$7.28(3 \mathrm{H}, \mathrm{m}, \mathrm{H}-7, \mathrm{H}-8, \mathrm{H}-9), 7.41-7.43(2 \mathrm{H}, \mathrm{m}, m-\mathrm{Ar}), 7.50$ (1H, d, J = 6.4 Hz, H-6), $7.74(2 \mathrm{H}$, $\mathrm{d}, J=8.6 \mathrm{~Hz}, o-\mathrm{Ar}), 8.47$ (1H, bs, NH). ${ }^{13} \mathrm{C}$ NMR $\delta: 34.94,42.33,64.67,124.69,124.80,127.60$, $127.97,128.34,129.28,132.31,137.66,141.25,143.25,162.67,172.34$.

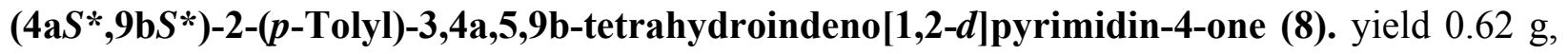
$77 \%$, mp 215-218 ${ }^{\circ} \mathrm{C}$. Anal. Calcd. for $\mathrm{C}_{18} \mathrm{H}_{16} \mathrm{~N}_{2} \mathrm{O}$ (276.13): C, 78.24; H, 5.84; N, 10.14. Found: 
C, 78.37; H, 5.98; N, 10.42. NMR data: ${ }^{1} \mathrm{H}$ NMR $\delta: 2.39$ (3H, s, $\left.\mathrm{CH}_{3}\right), 3.26-3.34$ (2H, m, H-5), $3.55(1 \mathrm{H}, \mathrm{dt}, J=11.9 \mathrm{~Hz}, 7.5 \mathrm{~Hz}, \mathrm{H}-4 \mathrm{a}), 5.50(1 \mathrm{H}, \mathrm{d}, J=7.5 \mathrm{~Hz}, \mathrm{H}-9 \mathrm{~b}), 7.23-7.26$ (5H, m, $m-\mathrm{Ar}$, H-7, H-8, H-9), 7.52 (1H, d, J=6.6 Hz, H-6), 7.66, (2H, d, J=8.1 Hz, o-Ar), 8.40 (1H, bs, NH).

${ }^{13} \mathrm{C}$ NMR $\delta: 21.62,34.87,42.45,64.53,124.71,124.74,126.46,127.9,128.18,129.69,131.07$, $140.19,141.79,143.53,167.34,172.35$.

\section{General procedure for the synthesis of 9 and 10}

A mixture of azetidinone $2(0.5 \mathrm{~g}, 3.14 \mathrm{mmol})$ and the corresponding lactim ether $(6.28 \mathrm{mmol})$ was kept at $150-160{ }^{\circ} \mathrm{C}$ for $8 \mathrm{~h}$. The excess of lactim ether was then evaporated off and the residue was dissolved in $\mathrm{Et}_{2} \mathrm{O}(20 \mathrm{ml})$, treated with charcoal, filtered and left to stand at $4{ }^{\circ} \mathrm{C}$. The product 9 or $\mathbf{1 0}$ was filtered off and recrystallized from $n$-hexane.

$\left(4 \mathrm{~b} S^{*}, 10 \mathrm{a} S^{*}\right)-6,7,8,9,10 \mathrm{a}, 11-\mathrm{Hexahydro-4bH}$-indeno[1,2-e]pyrido[1,2-a]pyrimidine (9). yield $0.44 \mathrm{~g}, 58 \%$, mp $47-49{ }^{\circ} \mathrm{C}$. Anal. Calcd. for $\mathrm{C}_{15} \mathrm{H}_{16} \mathrm{~N}_{2} \mathrm{O}$ (240.31): C, 74.97; H, 6.71; N, 11.66. Found: C, 75.21; H, 6.83; N, 11.72. NMR data: ${ }^{1} \mathrm{H}$ NMR $\delta: 1.71-1.83$ (4H, m, H-6, H-7), 2.49-2.64 (2H, m, H-8), 3.21-3.30 (2H, m, H-9), 3.39-3.47 (1H, m, H-11), 3.58-3.65 (1H, m, H11), 3.70-3.78 (1H, m, H-10a), 5.14 (1H, d, J=7.5 Hz, H-4b) 7.20-7.26 (3H, m, H-1, H-2, H-3), $7.52(1 \mathrm{H}, \mathrm{m}, \mathrm{H}-4) .{ }^{13} \mathrm{C}$ NMR $\delta: 19.77,22.32,31.96,35.50,40.83,42.70,62.36,124.39,124.50$, $127.25,127.84,140.34,143.66,151.63,170.80$.

$\left(4 \mathrm{~b} S^{*}, 11 \mathrm{a} S^{*}\right)-4 \mathrm{~b}, 6,7,8,9,10,11 \mathrm{a}, 12-O c t a h y d r o i n d e n o[1,2-e]$ azepino[1,2-a]pyrimidine (10). yield $0.42 \mathrm{~g}, 53 \%$, mp $70-72{ }^{\circ} \mathrm{C}$. Anal. Calcd. for $\mathrm{C}_{16} \mathrm{H}_{18} \mathrm{~N}_{2} \mathrm{O}$ (254.34): C, 75.56; H, 7.13; N, 11.01. Found: C, 75.63; H, 7.37; N, 11.29. NMR data: ${ }^{1} \mathrm{H}$ NMR $\delta: 1.54-1.71$ (4H, m, H-6, H-7), 1.71-1.84 (2H, m, H-8), 2.61-2.68 (2H, m, H-9), 3.19 (1H, dt, J=5.1, 7.8 Hz, H-11a), 3.26 (1H, $\mathrm{dd}, J=15.2,7.6 \mathrm{~Hz}, \mathrm{H}-10), 3.37(1 \mathrm{H}, \mathrm{dd}, J=15.2,5.1 \mathrm{~Hz}, \mathrm{H}-10), 3.80(1 \mathrm{H}, \mathrm{dd}, J=13.6,5.1 \mathrm{~Hz}$, H-12), 3.90 (1H, dd, $J=15.2,5.2 \mathrm{~Hz}, \mathrm{H}-12), 5.14$ (1H, d, $J=7.9 \mathrm{~Hz}, \mathrm{H}-4 \mathrm{~b}), 7.20-7.26$ (3H, m, $\mathrm{H}-1, \mathrm{H}-2, \mathrm{H}-3), 7.52$ (1H, m, H-4). ${ }^{13} \mathrm{C} \mathrm{NMR} \delta: 26.23,28.98,29.21,35.62,37.21,41.77,42.22$, $62.38,124.28,124.46,127.25,127.80,140.39,143.64,156.41,170.76$.

Ethyl cis-1-(4-chlorobenzylideneamino)indane-2-carboxylate (11). To a solution of amino ester 3 (1.54 g, $7.51 \mathrm{mmol})$ in $20 \mathrm{ml}$ absolute $\mathrm{MeOH}$, an equivalent amount of $p$ chlorobenzaldehyde was added, and the mixture was left to stand at ambient temperature for $4 \mathrm{~h}$. Crystalline product 11 was then filtered off: yield $2.4 \mathrm{~g}, 98 \%, \mathrm{mp} 110-11{ }^{\circ} \mathrm{C}$. Anal. Calcd. for $\mathrm{C}_{19} \mathrm{H}_{18} \mathrm{ClNO}_{2}$ (327.10): C, 69.62; H, 5.53; Cl, 10.82; N, 4.27. Found: C, 69.85; H, 5.69; Cl, 11.13; N, 4.52. NMR data: ${ }^{1} \mathrm{H}$ NMR $\delta: 1.10\left(3 \mathrm{H}, \mathrm{t}, J=7.1 \mathrm{~Hz}, \mathrm{CH}_{2} \mathrm{CH}_{3}\right), 3.11(1 \mathrm{H}, \mathrm{dd}, \mathrm{J}=15.6$ $\mathrm{Hz}, 7.9 \mathrm{~Hz}, \mathrm{H}-3), 3.62$ (1H, dt, $J=8.7,7.8 \mathrm{~Hz}, \mathrm{H}-2), 3.73$ (1H, dd, $J=15.6 \mathrm{~Hz}, 9.3 \mathrm{~Hz}, \mathrm{H}-3)$, 4.00-4.14 (2H, m, $\left.\mathrm{CH}_{2} \mathrm{CH}_{3}\right), 5.09(1 \mathrm{H}, \mathrm{d}, J=7.0 \mathrm{~Hz}, \mathrm{H}-1), 7.12-7.21(2 \mathrm{H}, \mathrm{m}, \mathrm{H}-4, \mathrm{H}-5), 7.26$ (1H, td, $J=7.4 \mathrm{~Hz}, 1.2 \mathrm{~Hz}, \mathrm{H}-6), 7.32$ (3H, d, $J=8.4 \mathrm{~Hz}, m-\mathrm{Ar}, \mathrm{H}-7), 7.63$ (2H, d, $J=8.4 \mathrm{~Hz}$, $o-\mathrm{Ar}), 8.34(1 \mathrm{H}, \mathrm{s}, \mathrm{CH}=\mathrm{N}) .{ }^{13} \mathrm{C} \mathrm{NMR} \delta: 14.79,33.51,51.02,60.79,76.18,125.18,125.45$, $127.23,128.93,129.18,130.03,134.89,137.16,142.21,143.15,159.46,172.34$.

$\left(2 S^{*}, 4 \mathrm{a} S^{*}, 9 \mathrm{~b} S^{*}\right)-2-(4-C h l o r o p h e n y l)-1,2,3,4 \mathrm{a}, 5,5 \mathrm{a}, 9 \mathrm{a}, 9 \mathrm{~b}-0 \mathrm{ctahydroindeno}[1,2-a] p y r i m i d i n-$

4-one (12). Compound $2(0.7 \mathrm{~g}, 2.1 \mathrm{mmol})$ was left to stand in room temperature for 5 days with a $\mathrm{MeOH}$ solution of $\mathrm{NH}_{3}(50 \mathrm{ml}, 25 \%)$, after which the solvent was evaporated off. The crude 
product was a mixture of the diastereomers (2:1), which was recrystallized from EtOAc-EtOH to give the major diastereomer: yield $0.35 \mathrm{~g}, 55 \%$, mp $175-178{ }^{\circ} \mathrm{C}$. Anal. Calcd. for $\mathrm{C}_{17} \mathrm{H}_{15} \mathrm{ClN}_{2} \mathrm{O}$ (298.77): C, 68.34; H, 5.06; Cl, 11.87; N, 9.38. Found: C, 68.51; H, 5.33; Cl, 11.92; N, 9.51. NMR data: ${ }^{1} \mathrm{H}$ NMR $\delta: 1.50$ (1H, s, NH-1), $3.18(1 \mathrm{H}, \mathrm{dt}, J=6.9 \mathrm{~Hz}, 9.5 \mathrm{~Hz}, \mathrm{H}-4 \mathrm{a}), 3.30$ (1H, dd, $J=16.4 \mathrm{~Hz}, 9.1 \mathrm{~Hz}, \mathrm{H}-5), 3.48$ (1H, dd, $J=16.4 \mathrm{~Hz}, 9.8 \mathrm{~Hz}, \mathrm{H}-5), 4.68$ (1H, t, $J=7.6 \mathrm{~Hz}, \mathrm{H}-$ 9b), 5.46 (1H, d, J = 9.0 Hz, H-2), 6.28 (1H, s, NH-3), 7.19-7.30 (3H, m, H-7, H-8, H-9), 7.35$7.42(5 \mathrm{H}, \mathrm{m}, \mathrm{H}-6, \mathrm{Ar}) .{ }^{13} \mathrm{C} \mathrm{NMR} \delta: 35.46,43.66,61.87,70.36,70.45,125.47,125.83,127.63$, $128.27,129.43,129.69,135.69,138.12,142.03,173.69$.

$\left(2 S^{*}, 4 \mathrm{a} S^{*}, 9 \mathrm{~b} S^{*}\right)-3-M e t h y l-2-(4-c h l o r o p h e n y l)-1,2,3,4 \mathrm{a}, 5,5 \mathrm{a}, 9 \mathrm{a}, 9 \mathrm{~b}-o c t a h y d r o i n d e n o[1,2-$

a]pyrimidin-4-one (13). Compound $2(0.7 \mathrm{~g}, 2.1 \mathrm{mmol})$ was left to stand in room temperature for 5 days with a $\mathrm{MeOH}$ solution of $\mathrm{MeNH}_{2}(50 \mathrm{ml}, 40 \%)$ and the solvent was evaporated off. The crude product was a mixture of the diastereomers $(3: 1)$, which was recrystallized from EtOAc-EtOH to give the major diastereomer: yield $0.37 \mathrm{~g}, 56 \%, \mathrm{mp} 168-172{ }^{\circ} \mathrm{C}$. Anal. Calcd. for $\mathrm{C}_{18} \mathrm{H}_{17} \mathrm{ClN}_{2} \mathrm{O}$ (312.80): C, 69.12; H, 5.48; Cl, 11,33; N, 8.96. Found: C, 69.31; H, 5.53; Cl, 11.47; N, 8.69. NMR data: ${ }^{1} \mathrm{H}$ NMR $\delta: 2.16(1 \mathrm{H}, \mathrm{bs}, \mathrm{NH}-1), 2.90\left(3 \mathrm{H}, \mathrm{s}, \mathrm{CH}_{3}\right), 3.11(1 \mathrm{H}, \mathrm{dt}$, $J=9.7 \mathrm{~Hz}, 7.3 \mathrm{~Hz}, \mathrm{H}-4 \mathrm{a}), 3.33(1 \mathrm{H}, \mathrm{dd}, J=16.7,7.3 \mathrm{~Hz}, \mathrm{H}-5), 3.42$, (1H, dd, $J=16.7,9.8 \mathrm{~Hz}$, H-5), 4.35 (1H, d, J = 7.1 Hz, H-9b), 5.26 (1H, s, H-2), 7.18-7.26 (4H, m, H-6, H-7, H-8, H-9), $7.32(2 \mathrm{H}, \mathrm{d}, J=8.4 \mathrm{~Hz}, m-\mathrm{Ar}), 7.41(2 \mathrm{H}, \mathrm{dt}, J=8.4 \mathrm{~Hz}, 1.8 \mathrm{~Hz}, o-\mathrm{Ar}) .{ }^{13} \mathrm{C}$ NMR $\delta: 32.81,35.59$, $43.78,57.52$, 74.94, 125.12, 125.19, 127.22, 128.70, 129.25, 129.67, 134.61, 137.12, 140.98, $142.19,171.81$.

\section{General procedure for the preparation of urea and thiourea compounds 14a-f}

To a magnetically stirred toluene solution of amino ester base 3 ( $0.5 \mathrm{~g}, 2.43 \mathrm{mmol}$ in $20 \mathrm{ml})$, one equivalent of the appropriate isocyanate or isothiocyanate in toluene $(20 \mathrm{ml})$ was added dropwise. The mixture was left to stand overnight at ambient temperature. The crystalline products were separated by filtration and recrystallized from EtOAc-MeOH.

cis- $\boldsymbol{N}$-Phenyl- $\boldsymbol{N}^{\prime}$-(2-ethoxycarbonylindanyl)urea (14a). yield $0.63 \mathrm{~g}, 81 \%$, mp 193-194 ${ }^{\circ} \mathrm{C}$. Anal. Calcd. for $\mathrm{C}_{19} \mathrm{H}_{20} \mathrm{~N}_{2} \mathrm{O}_{3}$ (324.38): C, 70.35; H, 6.21; N, 8.64. Found: C, 70.48; H, 6.48; N, 8.54. NMR data: ${ }^{1} \mathrm{H}$ NMR (400 MHz, $\left.\mathrm{CDCl}_{3}\right) \delta: 1.19\left(3 \mathrm{H}, \mathrm{t}, J=7.1 \mathrm{~Hz}, \mathrm{CH}_{2} \mathrm{CH}_{3}\right), 3.12(1 \mathrm{H}$, dd, $J=16.3 \mathrm{~Hz}, 8.5 \mathrm{~Hz}, \mathrm{H}-3), 3.29$ (1H, dd, $J=16.3 \mathrm{~Hz}, 5.5 \mathrm{~Hz}, \mathrm{H}-3), 3.62$ (1H, dt, $J=5.5,8.2$ $\mathrm{Hz}, \mathrm{H}-2), 4.06\left(2 \mathrm{H}, \mathrm{q}, J=7.14 \mathrm{~Hz}, \mathrm{CH}_{2} \mathrm{CH}_{3}\right), 5.56(1 \mathrm{H}, \mathrm{d}, J=7.9 \mathrm{~Hz}, \mathrm{NH}), 5.78(1 \mathrm{H}, \mathrm{dd}, J=$ 8.2, $7.9 \mathrm{~Hz}, \mathrm{H}-1), 6.66(1 \mathrm{H}, \mathrm{bs}, \mathrm{NHPh}), 7.02-7.08(1 \mathrm{H}, \mathrm{m}, p-\mathrm{Ph}), 7.15-7.34(8 \mathrm{H}, \mathrm{m}, o-\mathrm{Ph}, m-\mathrm{Ph}$, $\mathrm{H}-4, \mathrm{H}-5, \mathrm{H}-6, \mathrm{H}-7) .{ }^{13} \mathrm{C} \mathrm{NMR}\left(100 \mathrm{MHz}, \mathrm{CDCl}_{3}\right) \delta: 14.54,34.12,48.13,56.26,61.01,120.59$, $123.64,124.33,124.67,127.30,128.37,129.19,140.65,143.39,145.31,161.96,167.37$.

cis- $N$-Methyl- $N^{\prime}$-(2-ethoxycarbonylindanyl)thiourea (14b). yield $0.52 \mathrm{~g}, 77 \%$, mp 124$125^{\circ} \mathrm{C}$. Anal. Calcd. for $\mathrm{C}_{14} \mathrm{H}_{18} \mathrm{~N}_{2} \mathrm{O}_{2} \mathrm{~S}$ (278.38): C, 60.41; H, 6.52; N, 10.06. Found: C, 60.63; $\mathrm{H}, 6.58 ; \mathrm{N}, 10.21$. NMR data: ${ }^{1} \mathrm{H}$ NMR $\delta: 1.25\left(3 \mathrm{H}, \mathrm{t}, J=7.1 \mathrm{~Hz}, \mathrm{CH}_{2} \mathrm{CH}_{3}\right), 2.93(3 \mathrm{H}, \mathrm{s}$, $\left.\mathrm{CH}_{3}(\mathrm{Me})\right), 3.21(1 \mathrm{H}, \mathrm{dd}, J=8.2 \mathrm{~Hz}, 16.4 \mathrm{~Hz}, \mathrm{H}-3), 3.27(1 \mathrm{H}, \mathrm{dd}, J=4.8 \mathrm{~Hz}, 16.4 \mathrm{~Hz}, \mathrm{H}-3), 3.68$ $(1 \mathrm{H}, \mathrm{dt}, J=4.8,7.8 \mathrm{~Hz}, \mathrm{H}-2), 4.08\left(2 \mathrm{H}, \mathrm{q}, J=7.1 \mathrm{~Hz}, \mathrm{CH}_{2} \mathrm{CH}_{3}\right), 6.26(1 \mathrm{H}, \mathrm{bs}, \mathrm{NHMe}), 6.36(1 \mathrm{H}$, $\mathrm{dd}, J=8.0,7.8, \mathrm{H}-1), 6.73(1 \mathrm{H}, \mathrm{d}, J=8.0 \mathrm{~Hz}, \mathrm{NH}), 7.19-7.27$ (3H, m, H-4, H-5, H-6), 7.38 (1H, 
$\mathrm{d}, J=7.2 \mathrm{~Hz}, \mathrm{H}-7) .{ }^{13} \mathrm{C} \mathrm{NMR} \delta: 14.04,34.44,34.66,47.71,60.59,61.11,124.13,124.62$, $127.31,128.40,140.18,141.40,162.88,167.64$.

cis- $N$-Ethyl- $N^{\prime}$-(2-ethoxycarbonylindanyl)thiourea (14c). yield $0.56 \mathrm{~g}, 79 \%$, mp 122-123 ${ }^{\circ} \mathrm{C}$. Anal. Calcd. for: $\mathrm{C}_{15} \mathrm{H}_{20} \mathrm{~N}_{2} \mathrm{O}_{2} \mathrm{~S}(292.40) \mathrm{C}, 61.62 ; \mathrm{H}, 6.89 ; \mathrm{N}, 9.58$. Found: $\mathrm{C}, 61.69 ; \mathrm{H}, 7.18 ; \mathrm{N}$, 9.31. NMR data: ${ }^{1} \mathrm{H}$ NMR $\delta: 1.21\left(3 \mathrm{H}, \mathrm{t}, J=7.2 \mathrm{~Hz}, \mathrm{CH}_{2} \mathrm{CH}_{3}\right), 1.26(3 \mathrm{H}, \mathrm{t}, J=7.2 \mathrm{~Hz}$, $\left.\mathrm{CH}_{3} \mathrm{CH}_{2} \mathrm{NH}\right), 3.17-3.30(2 \mathrm{H}, \mathrm{m}, \mathrm{H}-3), 3.34\left(2 \mathrm{H}, \mathrm{bs}, \mathrm{CH}_{3} \mathrm{CH}_{2} \mathrm{NH}\right) 3.68(1 \mathrm{H}, \mathrm{dt}, J=4.9 \mathrm{~Hz}$, $7.7 \mathrm{~Hz}, \mathrm{H}-2), 4.08\left(2 \mathrm{H}, \mathrm{q}, J=7.1 \mathrm{~Hz}, \mathrm{CH}_{2} \mathrm{CH}_{3}\right), 6.14\left(1 \mathrm{H}, \mathrm{bs}, \mathrm{CH}_{3} \mathrm{CH}_{2} \mathrm{NH}\right), 6.38(1 \mathrm{H}, \mathrm{t}, J=7.8$ $\mathrm{Hz}, \mathrm{H}-1), 6.73$ (1H, d, $J=8.2 \mathrm{~Hz}, \mathrm{NH}), 7.19-7.26$ (3H, m, H-4, H-5, H-6) 7.37 (1H, d, $J=7.2$ $\mathrm{Hz}, \mathrm{H}-7) .{ }^{13} \mathrm{C}$ NMR $\delta: 13.97,14.54,34.69,47.66,48.29,60.52,61.09,124.07,124.61,127.28$, $128.34,140.11,141.44,174.36,181.72$.

cis- $\boldsymbol{N}$-Phenyl- $\boldsymbol{N}^{\prime}$-(2-ethoxycarbonylindanyl)thiourea (14d). yield $0.62 \mathrm{~g}, 75 \%$, mp 169 $170{ }^{\circ} \mathrm{C}$. Anal. Calcd. for $\mathrm{C}_{19} \mathrm{H}_{20} \mathrm{~N}_{2} \mathrm{O}_{2} \mathrm{~S}$ (340.45): C, 67.03; H, 5.92; N, 8.23. Found: C, 67.31; H, 5.78; N, 8.55. NMR data: ${ }^{1} \mathrm{H}$ NMR $\delta: 1.18\left(3 \mathrm{H}, \mathrm{t}, J=7.2 \mathrm{~Hz}, \mathrm{CH}_{2} \mathrm{CH}_{3}\right), 3.20(2 \mathrm{H}, \mathrm{d}, J=6.3 \mathrm{~Hz}$, H-3), $3.69(1 \mathrm{H}, \mathrm{dt}, J=7.3,6.3 \mathrm{~Hz}, \mathrm{H}-2), 3.99-4.07\left(2 \mathrm{H}, \mathrm{m}, \mathrm{CH}_{2} \mathrm{CH}_{3}\right), 6.49(1 \mathrm{H}, \mathrm{t}, J=9.1,7.3$ $\mathrm{Hz}, \mathrm{H}-1), 7.04$ (1H, d, $J=9.1 \mathrm{~Hz}, \mathrm{NH}), 7.16-7.27$ (6H, m, p-Ph, m-Ph, H-4, H-5, H-6), 7.31-7.34 $(1 \mathrm{H}, \mathrm{m}, \mathrm{H}-7), 7.35-7.41$ (2H, m, o-Ph), 8.09 (1H, bs, NHPh). ${ }^{13} \mathrm{C}$ NMR $\delta: 13.99,34.76,47.70$, $60.94,61.15,123.82,124.45,124.91,127.16,127.27,128.35,130.04,135.84,140.20,140.94$, $173.57,180.58$.

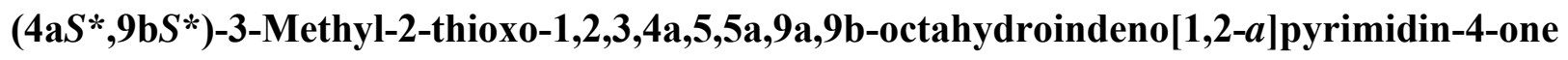
(15). Thiourea derivative 14b ( $0.70 \mathrm{~g}, 2.51 \mathrm{mmol}$ ) was dissolved in EtOH containing 22\% dry $\mathrm{HCl}(20 \mathrm{ml})$ and the solution was refluxed for $12 \mathrm{~h}$. After standing overnight, the crystalline product obtained was separated by filtration and recrystallized from EtOH. Yield $0.29 \mathrm{~g}, 50 \%$, mp 169-170 ${ }^{\circ} \mathrm{C}$. Anal. Calcd. for $\mathrm{C}_{12} \mathrm{H}_{12} \mathrm{~N}_{2} \mathrm{O}_{2} \mathrm{~S}$ (232.31): C, 62.04; H, 5.21; N, 12.06. Found: C, 62.32; H, 5.48; N, 12.23. NMR data: ${ }^{1} \mathrm{H}$ NMR $\delta: 3.28-3.37(1 \mathrm{H}, \mathrm{m}, \mathrm{H}-4 \mathrm{a}), 3.45-3.53$ (2H, m, H5), $3.54\left(3 \mathrm{H}, \mathrm{s}, \mathrm{CH}_{3}\right), 4.95$ (1H, dd, $\left.J=7.2,3.2 \mathrm{~Hz}, \mathrm{H}-9 \mathrm{~b}\right), 7.27-7.40$ (4H, m, H-6, H-7, H-8, H9). ${ }^{13} \mathrm{C}$ NMR $\delta: 33.59,35.13,43.59,57.12,123.71,125.28,127.77,129.36,139.53,140.65$, $168.84,181.18$.

1H-Indene-2-carboxylic acid (16). Thiourea derivative 14a, 14c or 14d (2.51 mmol) was dissolved in EtOH containing 22\% dry $\mathrm{HCl}(20 \mathrm{ml})$ and the solution was refluxed for $12 \mathrm{~h}$. After standing overnight, the crystalline product obtained was separated by filtration. Yield 48-53\%, mp 230-232 (decomp) ${ }^{\circ} \mathrm{C}$, lit $^{19} \mathrm{mp} \mathrm{234-236}{ }^{\circ} \mathrm{C},{ }^{1} \mathrm{H}$ NMR $\delta: 3.73(2 \mathrm{H}, \mathrm{d}, J=1.7 \mathrm{~Hz}, \mathrm{H}-1), 7.33-$ 7.40 (2H, m, H-6, H-7), 7.52-7.58 (2H, m, H-4, H-5), 7.87 (1H, t, J=1.7 Hz, H-3). ${ }^{13} \mathrm{C}$ NMR $\delta$ : $38.19,123.72,124.38,124,79,127.0,128.07,136.21,136.61,143.54,169.42 . \mathrm{MS} \mathrm{m} / \mathrm{z}$ (r.i.) 160 (65), 142 (3), 132 (6), 115 (100), 103 (3), 89 (8), 77 (4), 63 (9), 57 (3), 51 (2), 39 (3). Exact mass calculated for $\mathrm{C}_{10} \mathrm{H}_{8} \mathrm{O}_{2}: 160.05243$. Found: 160.05258 .

\section{$\left(4 \mathrm{a} S *, 6 \mathrm{a} S^{*}, 11 \mathrm{~b} S^{*}\right)-4 \mathrm{~b}, 5$-Dimethyl-4b,6,6a,7,11b,12-hexahydro-5H-indeno[1,2-e]pyrimido-}

[1,2-a]isoindole (19). To a solution of diamine base $5(0.6 \mathrm{~g}, 3.4 \mathrm{mmol})$ in $20 \mathrm{ml}$ absolute toluene, an equivalent amount of 2-carboxybenzaldehyde $(0.51 \mathrm{~g}, 3.4 \mathrm{mmol})$ was added, and the mixture was refluxed for $5 \mathrm{~h}$. The solvent was evaporated off and the residue was crystallized from $i \mathrm{Pr}_{2} \mathrm{O}$ and then recrystallized from $i \mathrm{Pr}_{2} \mathrm{O}$-EtOAc. Yield $0.64 \mathrm{~g}, 65 \%$, mp $150-153{ }^{\circ} \mathrm{C}$. Anal. 
Calcd. for $\mathrm{C}_{19} \mathrm{H}_{18} \mathrm{~N}_{2} \mathrm{O}$ (290.37): C, 78.59; H, 6.25; N, 9.65. Found: C, 78.71; H, 6.32; N, 9.73. NMR data: ${ }^{1} \mathrm{H}$ NMR $\delta: 2.09\left(3 \mathrm{H}, \mathrm{s}, \mathrm{CH}_{3}\right), 2.52(1 \mathrm{H}, \mathrm{d}, J=15.9 \mathrm{~Hz}, \mathrm{H}-7), 2.84(1 \mathrm{H}, \mathrm{t}, J=12.4$ Hz, H-6), 2.91-3.00 (1H, m, H-6a), 3.06 (1H, dd, $J=12.4$ Hz, 5.3 Hz, H-6), 3.14 (1H, dd, $J=$ $15.9 \mathrm{~Hz}, 6.5 \mathrm{~Hz}, \mathrm{H}-7), 5.1$ (1H, s, H-4b), 5.96 (1H, d, J=6.6Hz, H-11b), 7.15-7.29 (4H, m, H-8, H-9, H-10, H-11), 7.48-7.59 (3H, m, H-2, H-3, H-4), 7.94 (1H, d, J=7.2 Hz, H-1). ${ }^{13} \mathrm{C}$ NMR $\delta$ : 30.29 , 34.06, 34.35, 55.72, 55.81, 73.05, 123.60, 123.87, 124.26, 125.45, 127.11, 127.93, 129.29, $129.47,131.54,133.02,140.84,141.71,167.29$.

$\left(3 \mathrm{a} R^{*}, 5 \mathrm{a} S^{*}, 10 \mathrm{~b} S^{*}\right)-3 \mathrm{a}, 4-D i m e t h y l-1,2,3 \mathrm{a}, 4,5,5 \mathrm{a}, 6,10 \mathrm{~b}-0 \mathrm{ctahydro-3H}$-indeno[1,2-e]pyrrolo[1,2-a]pyrimidine (22). To a solution of diamine base $5(0.4 \mathrm{~g}, 2.27 \mathrm{mmol})$ in $20 \mathrm{ml}$ absolute toluene, an equivalent amount of levulinic acid $(0.27 \mathrm{~g}, 2.27 \mathrm{mmol})$ was added, and the mixture was refluxed for $8 \mathrm{~h}$. The solvent was evaporated off and the residue was chromatographed on silica. Elution with EtOAc afforded the pentacyclic 21, as the only diastereomer. Yield $0.37 \mathrm{~g}$, 63\%, mp 93-94 ${ }^{\circ} \mathrm{C}$. Anal. Calcd. for $\mathrm{C}_{16} \mathrm{H}_{20} \mathrm{~N}_{2} \mathrm{O}$ (256.35): C, 74.97; H, 7.86; N, 10.93. Found: C, 74.74; H, 7.98; N, 10.72. NMR data: ${ }^{1} \mathrm{H}$ NMR $\delta: 0.9\left(3 \mathrm{H}, \mathrm{s}, \mathrm{CH}_{3}\right), 2.01(2 \mathrm{H}, \mathrm{dd}, J=9.6 \mathrm{~Hz}$, $4.6 \mathrm{~Hz}, \mathrm{H}-3), 2.18$ (3H, s, NCH 3$), 2.37$ (1H, t, J=12.1 Hz, H-5), 2.47-2.64 (3H, m, H-2, H-6), $2.66(1 \mathrm{H}, \mathrm{dd}, J=12.1,6.4 \mathrm{~Hz}, \mathrm{H}-5), 2.74-2.83(1 \mathrm{H}, \mathrm{m}, \mathrm{H}-5 \mathrm{a}), 3.04$ (1H, dd, $15.8 \mathrm{~Hz}, 6.7 \mathrm{~Hz}, \mathrm{H}-$ 6), $5.62(1 \mathrm{H}, \mathrm{d}, J=7.1 \mathrm{~Hz}, \mathrm{H}-10 \mathrm{~b}), 7.12-7.15$ (4H, m, H-7, H-8, H-9, H-10). ${ }^{13} \mathrm{C}$ NMR $\delta: 15.37$, 29.66, 34.35, 34.50, 36.09, 37.76, 51.39, 54.78, 77.35, 124.58, 125.09, 126.76, 127.42, 140.66, $141.31,173.33$.

\section{General procedure to react diamine 5 with aromatic aldehydes $23 \mathrm{a}-\mathrm{g}$}

To a suspension of diamine $\mathbf{5}(0.2 \mathrm{~g}, 1.13 \mathrm{mmol})$ in $20 \mathrm{ml}$ absolute $\mathrm{MeOH}$, an equivalent amount of aromatic aldehyde was added (liquid aldehydes were freshly distilled), and the mixture was allowed to stand at ambient temperature for 1 day. The solvent was then evaporated off and the evaporation was repeated after the addition of $10 \mathrm{ml}$ toluene. The crystalline products were filtered off and recrystallized from $i \mathrm{Pr}_{2} \mathrm{O}$-EtOAc. The oily product $\mathbf{2 3 b}$ was dried in a vacuum desiccator for $24 \mathrm{~h}$.

Table 1. Physical data on compounds 23a-g

\begin{tabular}{|c|c|c|c|c|c|c|}
\hline Compound & $\mathbf{M p}\left({ }^{\circ} \mathbf{C}\right)$ & $\begin{array}{c}\text { Yield } \\
(\%)\end{array}$ & Formula & M.W & $\begin{array}{c}\delta \rho \mathrm{N}- \\
\text { CHAr-N } \\
\operatorname{ring}(\mathrm{B}) \\
(J[\mathrm{~Hz}])\end{array}$ & $\begin{array}{c}\delta \rho \mathrm{N}- \\
\mathrm{CHAr}-\mathrm{N} \\
\operatorname{ring}(\mathrm{C}) \\
(J[\mathrm{~Hz}])\end{array}$ \\
\hline $23 a$ & $143-144$ & 72 & $\mathrm{C}_{18} \mathrm{H}_{19} \mathrm{~N}_{3} \mathrm{O}_{2}$ & 309.37 & $4.62(6.3)$ & $4.20(5.2)$ \\
\hline $23 b$ & oil & - & $\mathrm{C}_{18} \mathrm{H}_{19} \mathrm{BrN}_{2}$ & 343.28 & $4.62(6.2)$ & $4.19(5.2)$ \\
\hline $23 c$ & $103-104$ & 73 & $\mathrm{C}_{18} \mathrm{H}_{19} \mathrm{ClN}_{2}$ & 298.92 & $4.60(6.2)$ & $4.19(5.2)$ \\
\hline 23d & $78-82$ & 80 & $\mathrm{C}_{18} \mathrm{H}_{20} \mathrm{~N}_{2}$ & 264.38 & $4.62(6.2)$ & $4.21(5.2)$ \\
\hline $23 e$ & $90-93$ & 75 & $\mathrm{C}_{19} \mathrm{H}_{22} \mathrm{~N}_{2}$ & 278.40 & $4.60(6.2)$ & $4.20(5.2)$ \\
\hline $23 f$ & $95-97$ & 75 & $\mathrm{C}_{19} \mathrm{H}_{22} \mathrm{~N}_{2} \mathrm{O}$ & 294.40 & $4.61(6.2)$ & $4.20(5.1)$ \\
\hline $23 g$ & $118-120$ & 82 & $\mathrm{C}_{20} \mathrm{H}_{25} \mathrm{~N}_{3}$ & 307.44 & $4.61(5.5)$ & $4.20(0.57)$ \\
\hline
\end{tabular}




\section{Acknowledgements}

We thank Ms. Erika Horváth for her kind assistance in the synthetic work. The authors acknowledge receipt of OTKA grant T 034901.

\section{References}

1. (a) Juaristi, E. Enantioselective Synthesis of $\beta$-Amino Acids, Wiley-VCH: New York, 1997.

(b) Fülöp, F. Chem. Rev. 2001, 101, 2181.

2. Fülöp, F.; Bernáth, G.; Pihlaja, K. Adv. Heterocyclic Chem. 1998, 69, 349.

3. Krotko, D. G.; Fedotov, K. V.; Tolmachev, A. I. Dyes and Pigments 2005, 65, 183.

4. Campagna, F.; Palluotto, F.; Mascia, M. P.; Maciocco. E. Marra, C. Carotti, A.; Carrieri, A. Farmaco 2003, 58, 129.

5. Ooms, F.; Frederick, R.; Durant, F.; Petzer, J. P.; Castagnoli, N.; Van der Schyf, C. J.; Wouters, J. Bioorg. Med. Chem. Lett. 2003, 13, 69.

6. Van Emelen, K.; De Wit, T.; Hoornaert, J.; Compernolle, F. Tetrahedron 2002, 58, 4225.

7. Mignani, S.; Bohme, G. A.; Birraux, G.; Boireau, A.; Jimonet, P.; Damour, D.; GenevoisBorella, A.; Debono, M. W.; Pratt, J.; Vuilhorgne, M.; Wahl, F.; Stutzmann, J. M. Bioorg. Med. Chem. 2002, 10, 1627.

8. Butera, J. A.; Antane, S. A.; Hirth, B.; Lennox, J. R.; Sheldon, J. H.; Norton, N. W.; Warga, D.; Argentieri, T. M. Bioorg. Med. Chem. Lett. 2001, 11, 2093.

9. De Wit, T.; Van Emelen, K.; Maertens, F.; Hoornaert, G. J.; Compernolle, F. Tetrahedron Lett. 2001, 42, 4919.

10. Stutzmann, J. M.; Bohme, G. A.; Boireau, A.; Damour, D.; Debono, M. W.; GenevoisBorella, A.; Jimonet, P.; Pratt, J.; Randle, J. C. R.; Ribeill, Y.; Vuilhorgne, M.; Mignani, S. Bioorg. Med. Chem. Lett. 2001, 11, 1205.

11. Fülöp, F.; Palkó, M.; Kámán, J.; Lázár, L.; Sillanpää, R. Tetrahedron: Asymmetry 2000, 11, 417.

12. Csomós, P.; Bernáth, G.; Fülöp, F. Monats. Chem. 2002, 133, 1077.

13. Hesse, M. Ring Enlargement in Organic Chemistry, VCH Publisher: New York, 1991.

14. Lázár, L.; Fülöp, F. Eur. J. Org. Chem. 2003, 3025.

15. Lázár, L.; Kivelä, H.; Pihlaja, K.; Fülöp, F. Tetrahedron Lett. 2004, 45, 6199.

16. Szatmári, I.; Hetényi, A.; Lázár, L.; Fülöp, F. J. Heterocyclic Chem. 2004, 41, 367.

17. Ito, T.; Yamazaki, N.; Kibayashi, C. Synlett 2001, 10, 1506.

18. Göblyös, A.; Lázár, L.; Fülöp. F. Tetrahedron 2002, 58, 1011.

19. Blocher, A.; Zeller, K. P. Chem. Ber. 1994, 127, 551. 